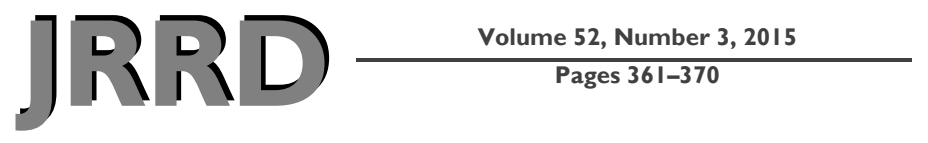

\title{
Effects of physical therapy delivery via home video telerehabilitation on functional and health-related quality of life outcomes
}

\author{
Charles E. Levy, MD; ${ }^{1-3^{*}}$ Erin Silverman, PhD; ${ }^{1-2,4}$, Huanguang Jia, PhD; ${ }^{1}$ Meghan Geiss, MS; ${ }^{5}$ David Omura, \\ DPT, MHA $^{1-2}$ \\ ${ }^{1}$ Center of Innovation on Disability and Rehabilitation Research and ${ }^{2}$ Physical Medicine and Rehabilitation Service, \\ North Florida/South Georgia Veterans Health System, Gainesville, FL; ${ }^{3}$ Department of Occupational Therapy, College \\ of Public Health and Health Professions, and ${ }^{4}$ Department of Physiological Sciences, College of Veterinary Medicine, \\ University of Florida, Gainesville, FL; ${ }^{5}$ Department of Counseling, Educational Psychology and Research, College of \\ Education, Health and Human Sciences, The University of Memphis, Memphis, TN
}

\begin{abstract}
This study examined functional outcomes, healthrelated quality of life (HRQoL), and satisfaction in a group of Veterans who received physical therapy via an in-home video telerehabilitation program, the Rural Veterans TeleRehabilitation Initiative (RVTRI). A retrospective, pre-post study design was used. Measures obtained from 26 Veterans who received physical therapy in the RVTRI program between February 22, 2010, and April 1, 2011, were analyzed. Outcomes were the Functional Independence Measure (FIM); Quick Disabilities of the Arm, Shoulder, and Hand measure; Montreal Cognitive Assessment (MoCA); and the 2-minute walk test (2MWT). HRQoL was assessed using the Veterans RAND 12-Item Health Survey (VR-12), and program satisfaction was evaluated using a telehealth satisfaction scale. Average length of participation was $99.2+/-43.3 \mathrm{~d}$ and Veterans, on average, received $15.2+/-6.0$ therapeutic sessions. Significant improvement was shown in the participants' FIM ( $p<0.001, r=0.63)$, MoCA $(p=0.01, r=0.44)$, 2MWT $(p=0.006, r=0.73)$, and VR-12 $(p=0.02, r=0.42)$. All Veterans reported satisfaction with their telerehabilitation experiences. Those enrolled in the RVTRI program avoided an average of 2,774.7 +/- 3,197.4 travel miles, $46.3+/-53.3 \mathrm{hr}$ of driving time, and $\$ 1,151.50+/-\$ 1,326.90$ in travel reimbursement. RVTRI provided an effective real-time, home-based, physical therapy.
\end{abstract}

Key words: functions, health-related quality of life, homebased physical therapy, mobility, physical therapy, satisfaction, telehealth, telerehabilitation, Veteran, Veteran rehabilitation.

\section{INTRODUCTION}

The mission of the Veterans Health Administration (VHA) of the Department of Veterans Affairs (VA) is to deliver uniform high-quality care to all Veterans, regardless of geography, distance, or economic circumstances. To meet this mission, the VHA must reach Veterans regardless of barriers to care provision, including long travel times and distances and expense. These barriers are magnified for rural Veterans with disabilities who require rehabilitation services. These individuals must invest

Abbreviations: $2 \mathrm{MWT}=$ 2-minute walk test; FIM = Functional Independence Measure; HRQoL = health-related quality of life; MoCA = Montreal Cognitive Assessment; MOVI = mobile video; NF/SG VHS = North Florida/South Georgia Veterans Health System; QuickDASH = Quick Disabilities of the Arm, Shoulder, and Hand measure; RVTRI = Rural Veterans TeleRehabilitation Initiative; SIP = Session Initiated Protocol; VA = Department of Veterans Affairs; VHA = Veterans Health Administration; VR-12 = Veterans RAND 12-Item Health Survey.

*Address all correspondence to Charles E. Levy, MD; PMRS (117) North Florida/South Georgia Veterans Health System, 1601 SW Archer Rd, Gainesville, FL 32608; 352374-6065; fax: 352-271-4540. Email: charles.levy@va.gov http://dx.doi.org/10.1682/JRRD.2014.10.0239 
additional time, thought, and resources in order to reach distant medical centers. Many rehabilitation protocols involve repeated therapy sessions, often two to five times weekly over weeks or months, resulting in additional physical, financial, and logistical hardships. In order to fulfill its promise, the VHA is actively attempting to address the gap in services for Veterans with limited access to traditional modes of treatment.

The VHA presently serves 3.3 million Veterans residing in rural localities. These individuals represent 41 percent of all Veterans enrolled in the VHA. Nearly 43 percent (2.27 million) of Veterans served by the VHA with a serviceconnected disability live in rural or highly rural areas [1]. Therefore, the VHA is looking to new technologies to facilitate access to healthcare for these individuals. As stated by W. Scott Gould, the former U.S. Deputy Secretary of Veterans Affairs, "We are investing more in telehealth technologies to make VA healthcare available to Veterans wherever they live. In FY [fiscal year] 2010, we invested \$121 million in telehealth. In FY2011, those investments will grow to $\$ 163$ million. By the end of FY2012, we expect to have doubled our present use of telehealth" [2]. Robert A. Petzel, the former Under Secretary for Health of the VA, has explicitly endorsed home telehealth technologies. In testimony before the House Committee on Veterans' Health on February 23, 2010, he stated, "Our increasing reliance on noninstitutional long-term care includes an investment in 2011 of $\$ 163$ million in home telehealth. Taking greater advantage of the latest technological advancements in healthcare delivery will allow us to more closely monitor the health status of Veterans and will greatly improve access to care. Telehealth will place specialized healthcare professionals in direct contact with patients using modern IT [information technology] tools" [3].

Telerehabilitation refers to the clinical application of consultative, preventative, diagnostic, and therapeutic services via two-way interactive telecommunication technologies [4-5]. Telerehabilitation is an alternative to usual-care outpatient rehabilitation services. It can also serve as an alternative to "homecare" rehabilitation, which requires the treating therapist or clinician to travel to the patient's home. By reducing or eliminating barriers relating to travel time and travel-related costs, telerehabilitation has the potential to improve access to rehabilitative care for stroke survivors [6-7]. Improving access to rehabilitative care may reduce disparities for stroke survivors and caregivers facing financial or transportation-related challenges. While research on telerehabilitation is limited, there is increasing evidence supporting the need for telerehabilitation services, the development of telerehabilitation interventions, and support for people with disabling conditions that potentially limit access to rehabilitation services [6-14].

The emerging field of video-based telerehabilitation allows therapists to deliver rehabilitative care to Veterans with physical, financial, and logistical barriers to healthcare providers and facilities [5]. Telerehabilitation has expanded dramatically in recent years as a result of advances in technology, increases in speed of telecommunication, and decreases in costs of computer hardware and software [6]. The scope of telerehabilitation includes direct therapeutic interventions, disease monitoring, coordination of care, patient and caregiver training and education, patient networking, and multidisciplinary professional consultation [15-16].

Veteran access to healthcare services is a topic of high interest and concern to both providers and researchers [6,17-20]. Numerous factors may interfere with patient access to healthcare, including distance, high travelrelated expenses, reduced numbers of healthcare providers within rural areas, transportation barriers, caregiver burden, attitude toward and perception of medical care providers, consumer knowledge, informal caregiver and/ or familial supports, and ethnic and cultural differences. Reduced access to healthcare contributes to increased morbidity and mortality, increased cost of treatment, and inappropriate use of emergency services [21-24]. Available technologies allow for rehabilitative services to be provided in real-time from providers' clinics to various recipients' locations such as home, community, health facilities, and/or work settings. While popular enthusiasm and capital investment in telerehabilitation continue to grow, very little is known regarding the efficacy of telerehabilitation or patients' overall evaluation and acceptance of telerehabilitation services [25]. A recent Cochran review concerning telerehabilitation services provided to patients during recovery from stroke concluded that sufficient data do not exist to support the effectiveness of telerehabilitation as a stand-alone replacement for traditional rehabilitative services for the restoration of activities of daily living, mobility, upper-limb function, healthrelated quality of life (HRQoL), patient satisfaction, or cost savings for patients receiving rehabilitative care following stroke [5]. The purpose of this study was to assess the functional outcomes, HRQoL, and satisfaction of a group of patients who participated in a VA telerehabilitation program. 


\section{METHODS}

\section{Study Design}

This was a retrospective, pre-post study using clinical data collected from the Rural Veterans TeleRehabilitation Initiative (RVTRI) clinical demonstration project between February 22, 2010, and April 1, 2011.

\section{Rural Veterans TeleRehabilitation Initiative Technology and Procedure}

The RVTRI, directed by the first author, is a clinical demonstration project established in 2009 with funding from the VHA Office of Rural Health. The RVTRI delivers real-time, patient-centered rehabilitation therapies such as occupational, physical, and recreational therapy, as well as psychological and nursing care directly to Veterans in their homes via Cisco E20 videophones (Cisco Systems Inc; San Jose, California). The Cisco E 20 is an in-home device that is similar to that previously reported by Tuerk et al. [26]. Once the determination is made that the Veteran is an appropriate candidate for telerehabilitation, the clinical service issues a consult request for the telecommunication equipment (in this case the Cisco E20 videophone) to prosthetics. Upon receiving the consult, the prosthetics service purchases and dispenses the device according to the directive of the original consult. In most cases, the videophones are delivered by mail to the home address of the Veteran who will be receiving treatment. Once the device is delivered, a clinical staff member is dispatched to the Veteran's place of residence in order to assist with establishing connectivity and providing the Veteran (and spouse or caregiver if appropriate) with in-depth instruction pertaining to the use of the device. The videophones connect to VA practitioners via a dedicated, secure, encrypted VA Internet network called the Veterans Affairs Central Office Expressway, which meets all Health Insurance Portability and Accountability Act privacy standards. Inside the VA, designated practitioners are able to complete the connection to the videophone through desktop computers configured with MOVI (mobile video) desktop video conferencing software and web cameras (Cisco Systems Inc). At scheduled appointment times, the Veteran can receive care remotely with high-quality, real-time video delivered over a secure, approved, encrypted Internet network.

\section{Technology Description}

The Cisco E20 is an IP (Internet protocol) voiceenabled phone that offers individual and group televideo and voice from one end point to another. The Cisco E20 offers an ultrawideband $20 \mathrm{kHz}$ speaker phone, acoustic echo cancellation, high-resolution camera with integrated privacy shutter, DVD quality (w448p video resolution), 10.6 in. wide format LCD display with WXGA resolution, and bandwidth Session Initiated Protocol (SIP) up to $1,152 \mathrm{kbps}$. Communication is safely transmitted through the firewall traversal through Cisco Video Communications Server Expressway. Each Cisco E20 is configured to connect to the gateway with a unique Web address.

The RVTRI network is made up of two sets of end points (video codecs): one set belongs to providers, and these end points reside on the VA Intranet, a private secure network behind the VA firewall; the other set of end points is issued to participating Veterans. The second set of end points resides on the Internet but is registered to the VA Expressway, hardware and software that provide a secure pathway through the VA firewall. Connections are made between Veteran and practitioner end points via stand-alone videophone or a secure VA computer with a web camera that utilizes an enterprise client license of Cisco MOVI, a SIP-based video application. Secure AES (Advanced Encryption Standard) encrypted video connections can be made from the Veterans' end points to the providers' end points via the VA Expressway using encryption that is embedded in each end point. No personal information is stored on the end points.

\section{Participants}

Veterans included in this report were referred for physical therapy services at the North Florida/South Georgia Veterans Health System (NF/SG VHS) and were seen face-to-face for the initial evaluation and treatment. If, at the time of initial evaluation, the Veteran expressed an interest in the RVTRI for follow-up care via videophone, he or she was screened in order to ensure that the following inclusionary criteria were met: (1) the Veteran consented to participate, (2) treatment was expected to require three or more therapist appointments, and (3) care did not require in-person treatment. For example, an individual needing hands-on joint mobilization from a physical therapist would not be eligible for the RVTRI since such care could not take place at a distance. However, Veterans receiving on-going management to ensure compliance with a home exercise program for low back pain could be candidates for the RVTRI. Participating Veterans were enrolled at physiatric mild traumatic brain injury 
clinics, spinal cord injury/mobility clinics, and general physical therapy clinics. At the therapist's discretion, the Veteran could be seen in person again. This might occur, for example, if the Veteran was coming to the medical center for an appointment with another provider and wished to take advantage of the opportunity to see his or her therapist in person or if the therapist wanted to reinforce a treatment plan in a true face-to-face manner.

A convenience sample of 26 Veterans who were enrolled in the RVTRI program between February 22, 2010, and April 1, 2011, served as participants in this retrospective study. The study cohort was 92.3 percent male, with 69.2 percent aged 50 to $64 \mathrm{yr}$ and 80.8 percent diagnosed with musculoskeletal disorders.

\section{Outcome Measures}

\section{Functional Independence Measure}

The Functional Independence Measure (FIM) is commonly used to assess patients' physical and cognitive independence while indicating the burden of rehabilitation care. This 18-item scale consists of 13 domains of physical function and 5 domains of cognitive function. Each item is scored from 1 (total dependence) to 7 (complete independence). Possible total scores range from 18 (lowest) to 126 (highest), with higher scores indicating greater independence [27].

\section{Quick Disabilities of the Arm, Shoulder, and Hand Measure}

The Quick Disabilities of the Arm, Shoulder, and Hand measure (QuickDASH) is an 11-item upper-limb physical function measurement tool. It provides clinicians with a brief and easily administered assessment of patients' upper-limb symptoms and level of disability. Dividing the completed response scores' sum by the number of responses yields an average value, which is transformed to a score out of 100 by subtracting 1 and multiplying by 25 . This final score is then comparable to other measures scaled on a 0-100 scale, with a higher score indicating greater disability [28].

\section{Montreal Cognitive Assessment}

The Montreal Cognitive Assessment (MoCA) is a screening tool for patients with mild cognitive dysfunction. This 30-point tool assesses 8 domains of cognitive function, including attention and concentration, executive functions, memory, language, visual constructional skills, conceptual thinking, calculations, and orientation. The maximum possible score on the MoCA is 30 points; a score of 26 or above is considered within normal range of functioning [29].

\section{2-Minute Walk Test}

The 2-minute walk test (2MWT) is a measure of ambulation that is reliable, valid, and relatively brief and easy to administer [30-31].

\section{Veterans RAND 12-Item Health Survey}

The Veterans RAND 12-Item Health Survey (VR-12) is a 12-item questionnaire with 8 principal physical and mental health domains, including general health perceptions, physical functioning, role limitations due to physical and emotional problems, bodily pain, energy fatigue, social functioning, and mental health. The 12 items can be summarized into 2 subscores measuring physical and mental health, as well as an overall summary score assessing patients' perceptions of their general HRQoL, with a higher score indicating better HRQoL [32].

\section{Satisfaction}

Patient satisfaction was evaluated using a telehealth satisfaction instrument designed for quality improvement monitoring for this study. The satisfaction measure consists of 13 items corresponding to aspects of the telerehabilitation experience. Twelve items use four- or five-point Likert rating scales; one item used a "yes" or "no" response.

With the exception of the satisfaction scale that was collected at discharged only, each of the previously referenced measurements were administered at two separate time points in the RVTRI program: at baseline, when a Veteran was first enrolled in the program, and at discharge, after the last appointment had taken place. Veterans were discharged from therapy either after goals were met or if the Veteran decided to withdraw from further treatment.

\section{Statistical Analysis}

All data were analyzed with SAS version 9.13 (SAS Institute Inc; Cary, North Carolina). Descriptive statistics were generated concerning Veterans' demographic characteristics, utilization variables, and satisfaction assessment. Each outcome measure was calculated at both baseline and discharge time points. Change in each outcome measure was calculated by subtracting the followup score from the baseline score. A Wilcoxon signed-rank test, also known as the Wilcoxon matched pairs test, was 
completed to compare the difference in repeated outcome measures on the study cohort [33]. Finally, we calculated the effect size of the Wilcoxon signed-rank test using the following formula: $r=\frac{Z}{\sqrt{N}}$, where $N$ is the total number of the samples.

\section{RESULTS}

This retrospective analytical study included 26 participants of the RVTRI, of whom 24 (92.3\%) were men, 69.2 percent were $64 \mathrm{yr}$ of age or less, and 57.7 percent lived in rural or highly rural area ZIP codes. Although the participants carried a variety of medical diagnoses, 80.8 percent were referred with musculoskeletal disorders, while 19.2 percent were referred with a neurological diagnosis (multiple sclerosis and stroke). The physical therapist's diagnosis (reason for treatment) included debility, balance, and mobility impairments for four individuals with neurological diagnoses, while the rest were diagnosed with cervicalgia, mechanical low back pain, shoulder pain, or knee pain (Table 1).

On average, the Veterans were enrolled in physical therapy for $99.2 \pm 43.3 \mathrm{~d}$ and averaged a total $15.2 \pm 6.0$ sessions. Veterans participated in an average of $3.9 \pm 2.6$ face-to-face sessions, comprising roughly 26 percent of all therapy visits. On average, $11.3 \pm 5.9$ visits were con-

Table 1.

Patient characteristics $(N=26)$.

\begin{tabular}{lc}
\hline \multicolumn{1}{c}{ Characteristics } & Percent \\
\hline Sex: Male & 92.3 \\
Age & \\
$\leq 64 \mathrm{yr}$ & 69.2 \\
>64 yr & 30.8 \\
Patients by Home ZIP Codes & \\
$\quad$ Rural Patients & 57.7 \\
$\quad$ Nonrural Patients & 42.3 \\
Major Medical Diagnosis & \\
$\quad$ Musculoskeletal & 80.8 \\
Multiple Sclerosis & 15.4 \\
$\quad$ Stroke & 3.8 \\
Types of Physical Therapy Provided & \\
General Strengthening Program & 26.9 \\
Lumbar Stabilization Program & 38.5 \\
Scapular Stabilization & 34.6 \\
\hline \hline
\end{tabular}

ducted remotely via telerehabilitation, 74 percent of all therapy visits (Table 2).

On average, the roundtrip mileage from the Veterans' home residences to the local VA hospital was $179.8 \pm$ 182.7 miles. Treatments rendered through the RVTRI program saved patients an average total number of 2,774.7 \pm 3,197.4 miles and $46.3 \pm 53.3$ total hours driving. Additionally, VHA saved an average of $\$ 1.151 .50 \pm$ $\$ 1,326.90$ per Veteran in potential travel reimbursement (Table 2). These reported savings do not include VHA reimbursements for hotel stays and meals, nor do they include childcare costs or lost wages/work time for the Veterans served.

Table 3 shows the average scores of each outcome measurement at different study time points, $p$-values from our Wilcoxon signed-rank tests, and the $r$-scores from our effect size calculation. Veterans showed significant improvement in most outcome measures between baseline and discharge, with large effect sizes for the FIM $(p<0.001, r=0.63)$ and the 2MWT $(p=0.006, r=0.73)$ and medium effect sizes for the MoCA ( $p=0.01, r=$ $0.44)$ and the VR-12 ( $p=0.02, r=0.42)$. Restoration of upper-limb function, as assessed by the QuickDASH,

Table 2.

Patient visits, travel, and potential cost avoidance.

\begin{tabular}{|c|c|}
\hline $\begin{array}{c}\text { Visit, Travel, And Potential Cost } \\
\text { Avoidance }\end{array}$ & Mean \pm SD \\
\hline 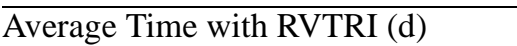 & $99.2 \pm 43.3$ \\
\hline $\begin{array}{l}\text { Average Number of Sessions } \\
\text { Received }\end{array}$ & $15.2 \pm 6.0$ \\
\hline $\begin{array}{l}\text { Average Number of Telerehabilitation } \\
\text { Sessions Received }\end{array}$ & $11.3 \pm 5.9$ \\
\hline $\begin{array}{l}\text { Average Number of Face-To-Face } \\
\text { Sessions Received }\end{array}$ & $3.9 \pm 2.6$ \\
\hline $\begin{array}{l}\text { Average Roundtrip Miles Per Visit } \\
\text { Saved* }\end{array}$ & $179.8 \pm 182.7$ \\
\hline $\begin{array}{l}\text { Average Total Number of Miles } \\
\text { Saved }^{\dagger}\end{array}$ & $2,774.7 \pm 3,197.4$ \\
\hline Average Total Driving Hours Saved & $46.3 \pm 53.3$ \\
\hline Average Travel Expense Saved $(\$)^{\ddagger}$ & $1,151.50 \pm 1,326.90$ \\
\hline \multicolumn{2}{|c|}{$\begin{array}{l}{ }^{*} \text { Roundtrip mileage from patients’ home residence to closest local Department } \\
\text { of Veterans Affairs (VA) hospital for each visit. } \\
{ }^{\circ} \text { Roundtrip miles per visit } \times \text { number of telerehabilitation sessions received } \\
\text { through RVTRI program. } \\
{ }^{\ddagger} \text { Total number of miles saved } \times \$ 0.415 \text { (per mile travel reimbursement rate to } \\
\text { eligible Veterans by VA for } 2010-2011 \text { ). } \\
\text { RVTRI = Rural Veterans TeleRehabilitation Initiative, SD = standard deviation. }\end{array}$} \\
\hline
\end{tabular}


improved from baseline to discharge. However, these results did not rise to the level of statistical significance (Table 3).

Table 4 provides data pertaining to patient satisfaction with various aspects of the RVTRI. Of 26 Veterans, 25 completed the survey. Of these, 92 percent (23/25) reported that they were able to connect with their physical therapist in 5 min or fewer. All patients reported being "satisfied" or "very satisfied" with the time spent with the therapist. Additional findings supported extremely high levels of satisfaction with the providers' personal manner, interactions with providers during the care, privacy, operation of telehealth equipment, and the audiovisual quality of the equipment. All but one (96\%) of the Veterans were either "satisfied" or "very satisfied" with their telehealth experience. All Veterans responded that they would use telehealth again for medical care (Table 4).

\section{DISCUSSION}

Telerehabilitation has expanded dramatically in recent years, both inside and outside the VHA. The rapid development and increasing speed of telecommunication technologies requires continuous study in order to establish the efficacy of such technologies. This study expands upon existing knowledge by assessing functional outcomes, HRQoL, and overall patient satisfaction of a group of Veterans who participated in the RVTRI, a real-time and patient-centered telerehabilitation program.

Table 3.

Results from Wilcoxon signed-rank tests and effect sizes.

\begin{tabular}{|c|c|c|c|c|c|}
\hline & Baseline & Discharge & Change Score & $p$-Value* & $r$ Score \\
\hline$\overline{\mathrm{VR}}-12(n=23)$ & $35.8 \pm 8.5$ & $39.7 \pm 9.8$ & $3.8 \pm 7.1$ & 0.02 & 0.42 \\
\hline FIM $(n=26)$ & $107.2 \pm 23.7$ & $114.7 \pm 15.7$ & $7.6 \pm 13.6$ & $<0.001$ & 0.63 \\
\hline Quick DASH $(n=19)$ & $40.0 \pm 19.4$ & $36.8 \pm 22.3$ & $-3.1 \pm 14.4$ & 0.35 & -0.22 \\
\hline $\operatorname{MoCA}(n=16)$ & $23.7 \pm 3.8$ & $26.2 \pm 4.4$ & $2.5 \pm 3.9$ & 0.01 & 0.44 \\
\hline $2 \mathrm{MWT}(n=13)$ & $319.4 \pm 182.2$ & $392.9 \pm 240.7$ & $73.5 \pm 86.7$ & 0.006 & 0.73 \\
\hline
\end{tabular}

${ }^{*} p$-Values were results from Wilcoxon signed-rank tests comparing baseline score and follow-up score for each measurement. Bold indicates significance. 2MWT = 2-minute walk test, FIM = Functional Independence Measure, MoCA = Montreal Cognitive Assessment, VR-12 = Veterans RAND 12-Item Health Survey.

Table 4.

Patient satisfaction. Number of respondents for satisfaction survey was 25 for all items except item number 12, which had 24 respondents.

\begin{tabular}{|c|c|c|c|c|c|}
\hline \multirow{2}{*}{ Satisfaction Item and Response Category } & \multicolumn{5}{|c|}{ Level of Satisfaction, $n(\%)$} \\
\hline & 1 & 2 & 3 & 4 & 5 \\
\hline 1. Length of time for 1st appointment ${ }^{*}$ & $0(0.0)$ & $0(0.0)$ & $11(44.0)$ & $14(56.0)$ & N/A \\
\hline 3. Time spent with your specialist ${ }^{*}$ & $0(0.0)$ & $0(0.0)$ & $3(12.0)$ & $22(88.0)$ & N/A \\
\hline 4. Your providers’ personal manner ${ }^{\ddagger}$ & $0(0.0)$ & $0(0.0)$ & $1(4.0)$ & $2(8.0)$ & $22(88.0)$ \\
\hline 6. Your privacy was respected ${ }^{*}$ & $0(0.0)$ & $0(0.0)$ & $3(12.0)$ & $22(88.0)$ & N/A \\
\hline 8. Equipment operation was explained ${ }^{*}$ & $0(0.0)$ & $1(4.0)$ & $3(12.0)$ & $21(84.0)$ & N/A \\
\hline 9. Satisfied with video quality ${ }^{*}$ & $0(0.0)$ & $2(8.0)$ & $8(32.0)$ & $15(60.0)$ & N/A \\
\hline 10. Satisfied with audio quality ${ }^{*}$ & $0(0.0)$ & $2(8.0)$ & $6(24.0)$ & $17(68.0)$ & N/A \\
\hline 11. Understanding your specialist ${ }^{*}$ & $0(0.0)$ & $1(4.0)$ & $4(16.0)$ & $20(80.0)$ & N/A \\
\hline 12. Overall telehealth experience ${ }^{*}$ & $0(0.0)$ & $1(4.17)$ & $6(25.0)$ & $17(70.8)$ & N/A \\
\hline
\end{tabular}


Results of this investigation provide evidence of significant improvements in physical function, cognitive function, functional independence, and HRQoL through telerehabilitation. Measures of overall satisfaction with the RVTRI were very high and serve as an indirect measure of the quality of the program itself. Patient satisfaction is an important measure for ensuring the quality of telerehabilitation care. Measures of satisfaction may also relate to levels of patient motivation and compliance with prescribed treatment regimens [17].

Our study had several limitations. First, although the sample received the majority (74\%) of therapy sessions via in-home telehealth, approximately a quarter of therapy sessions were completed in a traditional, face-to-face format. Therefore, the present investigation more accurately reflects a mixed-method approach to rehabilitation. The relative influence of face-to-face versus telehealth therapy sessions on the reported outcome measures cannot be determined for this sample. Second, without a control group, it is not known what portion of the observed changes in outcome measures between baseline and discharge occurred as a result of natural recovery. Additionally, the small sample size precluded use of robust, risk-adjusted regression analyses. This study was a retrospective examination of an existing clinical demonstration project, not a prospective randomized trial. As such, patients were not selected on the basis of diagnosis or level of impairment and length of treatment was highly variable. Our study cohort consisted of a group of Veterans with a variety of primary medical conditions, including musculoskeletal disorders, multiple sclerosis, and stroke. Although we did not observe statistically significant improvements in the QuickDASH measure, it is likely that, because of the heterogeneity of our patient group, only a minority had significant upper-limb impairment at baseline. The outcome data only contain two time points (baseline and discharge), and we were unable to observe the effects of the intervention beyond the end of therapy.

\section{CONCLUSIONS}

Despite these limitations, our findings document significant and meaningful improvement in patients' physical and cognitive functioning as well as HRQoL. Use of telerehabilitation was associated with minimizing the time, expense, and inconvenience of receiving rehabilitative care, both for Veterans and the VHA. High levels of satisfaction demonstrate that the RVTRI was successful in fulfilling the patient-centered mission of the VHA. These results indicate that in-home video telerehabilitation is a promising potential alternative to standard face-to-face rehabilitative care. Although this was not a rigorously controlled clinical trial, the fact that an actual clinical practice was examined may suggest that the results more closely mirror what actually occurs in clinical treatment.

\section{ACKNOWLEDGMENTS}

\section{Author Contributions:}

Study concept and design: C. E. Levy.

Acquisition of data: C. E. Levy, M. Geiss, D. Omura.

Analysis and interpretation of data: C. E. Levy, E. Silverman, H. Jia.

Drafting of manuscript: C. E. Levy, H. Jia.

Critical revision of manuscript for important intellectual content:

C. E. Levy, E. Silverman, M. Geiss, D. Omura.

Statistical analysis: H. Jia.

Obtained funding: C. E. Levy.

Administrative, technical, or material support: C. E. Levy.

Study supervision: C. E. Levy, M. Geiss, D. Omura.

Financial Disclosures: The authors have declared that no competing interests exist.

Funding/Support: This material was based on work supported by the RVTRI, funded by the VA Office of Rural Health (Project X08-

FY12Q1-S3-P00058) to Dr. Charles Levy and supported by the NF/ SG VHS.

Additional Contributions: Meghan Geiss has earned her $\mathrm{PhD}$ since the study was performed.

Institutional Review: This study was approved by the Institutional Review Board at the University of Florida (IRB-01) and the Research and Development Committee of the NF/SG VHS at Gainesville, Florida. Participant Follow-Up: The authors have no plans to inform participants of publication of this study.

Disclaimer: The views and opinions expressed in this article reflect those of the authors and do not necessarily reflect those of the VA and its Office of Rural Health and NF/SG VHS.

\section{REFERENCES}

1. Office of Rural Health. Fact sheet: Information about the Office of Rural Health and rural Veterans. Washington (DC): Veterans Health Administration; 2012.

2. Remarks by Former Deputy Secretary W. Scott Gould. 2010 Healthcare Innovation Day, [Internet]. Washington (DC): Department of Veterans Affairs; 2010 [2010 Oct 12; cited 2013 Jan 12]. Available from: http://www.Va.Gov/opa/speeches/2010/10 12 2010.Asp 
3. Petzel RA. House Committee on Veterans Affairs. Statement of the Honorable Robert A. Petzel, MD. Washington (DC): Veterans Health Administration; 2010.

4. Wakeford L, Wittman PP, White MW, Schmeler MR; Commission on Practice. Telerehabilitation position paper. Am J Occup Ther. 2005;59(6):656-60. [PMID:16363187] http://dx.doi.org/10.5014/ajot.59.6.656

5. Laver KE, Schoene D, Crotty M, George S, Lannin NA, Sherrington C. Telerehabilitation services for stroke. Cochrane Database Syst Rev. 2013;12:CD010255. [PMID:24338496]

6. Dorsey ER, George BP, Leff B, Willis AW. The coming crisis: Obtaining care for the growing burden of neurodegenerative conditions. Neurology. 2013;80(21):1989-96. [PMID:23616157] http://dx.doi.org/10.1212/WNL.0b013e318293e2ce

7. Chumbler NR, Quigley P, Li X, Morey M, Rose D, Sanford J, Griffiths P, Hoenig H. Effects of telerehabilitation on physical function and disability for stroke patients: A randomized, controlled trial. Stroke. 2012;43(8):2168-74. [PMID:22627983] http://dx.doi.org/10.1161/STROKEAHA.111.646943

8. Chumbler NR, Rose DK, Griffiths P, Quigley P, McGeeHernandez N, Carlson KA, Vandenberg P, Morey MC, Sanford J, Hoenig H. Study protocol: home-based telehealth stroke care: a randomized trial for veterans. Trials. 2010; 11:74. [PMID:20591171] http://dx.doi.org/10.1186/1745-6215-11-74

9. Durfee W, Carey J, Nuckley D, Deng J. Design and implementation of a home stroke telerehabilitation system. Conf Proc IEEE Eng Med Biol Soc. 2009;2009:2422-25. [PMID:19965201]

10. Johansson T, Wild C. Telerehabilitation in stroke care-a systematic review. J Telemed Telecare. 2011;17(1):1-6. [PMID:21097560] http://dx.doi.org/10.1258/jtt.2010.100105

11. Langan J, Delave K, Phillips L, Pangilinan P, Brown SH. Home-based telerehabilitation shows improved upper limb function in adults with chronic stroke: A pilot study. J Rehabil Med. 2013;45(2):217-20. [PMID:23319181] http://dx.doi.org/10.2340/16501977-1115

12. Morales-Vidal S, Ruland S. Telemedicine in stroke care and rehabilitation. Top Stroke Rehabil. 2013;20(2):101-7. [PMID:23611850] http://dx.doi.org/10.1310/tsr2002-101

13. Perry JC, Ruiz-Ruano JA, Keller T. Telerehabilitation: Toward a cost-efficient platform for post-stroke neurorehabilitation. Proceedings of the IEEE International Conference on Rehabilitation Robotics; 2011 Jun 29-Jul 1; Zurich, Switzerland.

14. Saywell N, Vandal AC, Brown P, Hanger HC, Hale L, Mudge S, Milosavljevic S, Feigin V, Taylor D. Telerehabil- itation to improve outcomes for people with stroke: Study protocol for a randomised controlled trial. Trials. 2012; 13:233. [PMID:23216861]

http://dx.doi.org/10.1186/1745-6215-13-233

15. Theodoros D, Russell T. Telerehabilitation: Current perspectives. Stud Health Technol Inform. 2008;131:191-209. [PMID:18431862]

16. Durfee WK, Savard L, Weinstein S. Technical feasibility of teleassessments for rehabilitation. IEEE Trans Neural Syst Rehabil Eng. 2007;15(1):23-29. [PMID:17436872] http://dx.doi.org/10.1109/TNSRE.2007.891400

17. Clark L. Mexican-origin mothers' experiences using children's health care services. West J Nurs Res. 2002;24(2): 159-79. [PMID:11858347] http://dx.doi.org/10.1177/01939450222045815

18. Demiris G, Parker Oliver DR, Courtney KL, Porock D. Use of technology as a support mechanism for caregivers of hospice patients. J Palliat Care. 2005;21(4):303-9. [PMID:16483101]

19. Demiris G, Shigaki CL, Schopp LH. An evaluation framework for a rural home-based telerehabilitation network. J Med Syst. 2005;29(6):595-603. [PMID:16235812] http://dx.doi.org/10.1007/s10916-005-6127-z

20. Piette JD, Moos RH. The influence of distance on ambulatory care use, death, and readmission following a myocardial infarction. Health Serv Res. 1996;31(5):573-91. [PMID:8943991]

21. Chanussot-Deprez C, Contreras-Ruiz J. Telemedicine in wound care: A review. Adv Skin Wound Care. 2013;26(2): 78-82. [PMID:23337648] http://dx.doi.org/10.1097/01.ASW.0000426717.59326.5f

22. Isetta V, Lopez-Agustina C, Lopez-Bernal E, Amat M, Vila M, Valls C, Navajas D, Farre R. Cost-effectiveness of a new internet-based monitoring tool for neonatal postdischarge home care. J Med Internet Res. 2013;15(2):e38. [PMID:23419609] http://dx.doi.org/10.2196/jmir.2361

23. Morland LA, Raab M, Mackintosh MA, Rosen CS, Dismuke CE, Greene CJ, Frueh BC. Telemedicine: A costreducing means of delivering psychotherapy to rural combat veterans with PTSD. Telemed J E Health. 2013;19(10): 754-59. [PMID:23931729] http://dx.doi.org/10.1089/tmj.2012.0298

24. Wu O, Langhorne P. The challenge of acute-stroke management: Does telemedicine offer a solution? Int J Stroke. 2006;1(4):201-7. [PMID:18706017] http://dx.doi.org/10.1111/j.1747-4949.2006.00062.x

25. Levy CE, Cowper-Ripley D, Hoffman P, Jia H, Mann W, Omura D. Rural Veterans TeleRehabilitation Initiative: Interim progress report. PM R. 2011;3:S260. http://dx.doi.org/10.1016/j.pmrj.2011.08.285 
26. Tuerk PW, Yoder M, Ruggiero KJ, Gros DF, Acierno R. A pilot study of prolonged exposure therapy for posttraumatic stress disorder delivered via telehealth technology. J Trauma Stress. 2010;23(1):116-23. [PMID:20135675]

27. Beninato M, Gill-Body KM, Salles S, Stark PC, BlackSchaffer RM, Stein J. Determination of the minimal clinically important difference in the FIM instrument in patients with stroke. Arch Phys Med Rehabil. 2006;87(1):32-39. [PMID:16401435] http://dx.doi.org/10.1016/j.apmr.2005.08.130

28. Gummesson C, Ward MM, Atroshi I. The shortened disabilities of the arm, shoulder and hand questionnaire (QuickDASH): Validity and reliability based on responses within the full-length DASH. BMC Musculoskelet Disord. 2006;7:44. [PMID:16709254] http://dx.doi.org/10.1186/1471-2474-7-44

29. Nasreddine ZS, Phillips NA, Bédirian V, Charbonneau S, Whitehead V, Collin I, Cummings JL, Chertkow H. The Montreal Cognitive Assessment, MoCA: A brief screening tool for mild cognitive impairment. J Am Geriatr Soc. 2005;53(4):695-99. [PMID:15817019] http://dx.doi.org/10.1111/j.1532-5415.2005.53221.x

30. Kosak M, Smith T. Comparison of the 2-, 6-, and 12-minute walk tests in patients with stroke. J Rehabil Res Dev. 2005;42(1):103-7. [PMID:15742254]

31. Leung AS, Chan KK, Sykes K, Chan KS. Reliability, validity, and responsiveness of a 2-min walk test to assess exer- cise capacity of COPD patients. Chest. 2006;130(1):119-25. [PMID:16840391]

http://dx.doi.org/10.1378/chest.130.1.119

32. Selim AJ, Rogers W, Fleishman JA, Qian SX, Fincke BG, Rothendler JA, Kazis LE. Updated U.S. population standard for the Veterans RAND 12-item Health Survey (VR12). Qual Life Res. 2009;18(1):43-52. [PMID:19051059] http://dx.doi.org/10.1007/s11136-008-9418-2

33. Conover WJ. Practical nonparametric statistics. 2nd ed. New York (NY): Wiley; 1980.

Submitted for publication October 16, 2014. Accepted in revised form February 12, 2015.

This article and any supplementary material should be cited as follows:

Levy CE, Silverman E, Jia H, Geiss M, Omura D. Effects of physical therapy delivery via home video telerehabilitation on functional and health-related quality of life outcomes. J Rehabil Res Dev. 2015;52(3):361-70. http://dx.doi.org/10.1682/JRRD.2014.10.0239

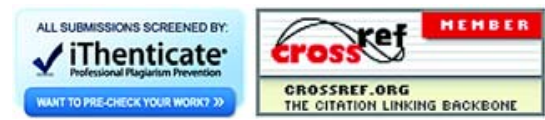


